\title{
Computing with Transiently Stable States
}

\author{
Mustafa C. Ozturk, Jose C. Principe \\ Electrical Engineering Department, University of Florida, Gainesville, FL 32611, USA
}

\begin{abstract}
Stability is an essential constraint in the design of linear dynamical systems. Similar stability restrictions on nonlinear dynamical systems, such as echo state network, have been enforced in order to use them for reliable computation. In this paper we will introduce a novel computational mode for nonlinear systems with sigmoidal nonlinearity, which does not require global stability. In this mode, although the autonomous system is unstable, the input signal forces the system dynamics to become "transiently stable". We demonstrate with a function approximation experiment that the transiently stable system can still do useful computation. We explain the principles of computation with the stability of local dynamics obtained from linearization of the system at the operating point.
\end{abstract}

\section{INTRODUCTION}

Linear filters are the simplest dynamical systems employed to process signals with temporal structure. They can be classified according to the impulse response, which fully characterizes the filter, as finite impulse response (FIR) and infinite impulse response (IIR) filters [1]. For the IIR case, stability becomes an essential constraint in the design. Without stability, the system response will diverge independent of the input signal. Global asymptotic stability of linear filters can simple be guaranteed by selecting the poles of the system in the proper region of the frequency domain, open left half plane for the continuous-time systems and inside the unit circle for the discrete-time systems [1].

Two important families of counterparts for the FIR and IIR filters in nonlinear dynamical systems are the time delay neural network (TDNN) and the recurrent neural network (RNN) [2]. Similar to the linear case, stability becomes an issue for recurrent nonlinear dynamical systems, although the definition of stability is no longer BIBO (bounded input bounded output) stability. In the echo state network (ESN), a recently proposed RNN type, this issue is addressed with a condition on the recurrent connection weights, the echo state condition [3]. The ESN is composed of two parts: a dynamical reservoir of recurrent processing elements and an instantaneous readout that reads the reservoir and produces the network output (see Fig.1) [3] [4]. The reservoir weights are fixed while the readout weights are adaptive. The echo state condition is defined in terms of the spectral radius of the reservoir's weight matrix $(|\mathbf{W}|<1)$. This condition is derived from the stability of the linearization of the system around zero equilibrium [3]. Echo state condition guarantees that the states are strongly coupled with the input signal not the initial conditions allowing the system to compute an input-output map.

In this paper, we introduce a new computational mode that emerges when we relax the echo state condition and allow the spectral radius to be slightly greater than 1 . In this mode, the autonomous system is not asymptotically stable since the spectral radius is greater than 1 . In other words, the states will not converge to zero, which is a natural equilibrium of the system, if they are initialized to a nonzero value. However, with the application of a sufficiently large constant input signal, the states will always converge to the same state value independent of the initial condition. Therefore, the system is asymptotically unstable with a zero input signal whereas sufficiently large signal makes the system asymptotically stable; hence the system is called "transiently stable". In transiently stable computation, the system response is determined by a competition between the system dynamics and the input dynamics. This mode can be appropriately called the "edge of chaos" as coined by Langton [8]. We will compare the states of ESN designed using echo state condition with the transiently stable ESN. We will also demonstrate with a function approximation experiment that the transiently stable ESN can also do useful computation but under a different computational mode where the stability of the local system dynamics is changing.

The principles of transiently stable computation will be explained using the local dynamics that is obtained by the linearization of the system around current operating point. We will see that the poles of the linearized system without the input signal move in and out of the unit circle in z-domain whereas with the application of the input signal, the poles will be brought inside the unit circle stabilizing the system.

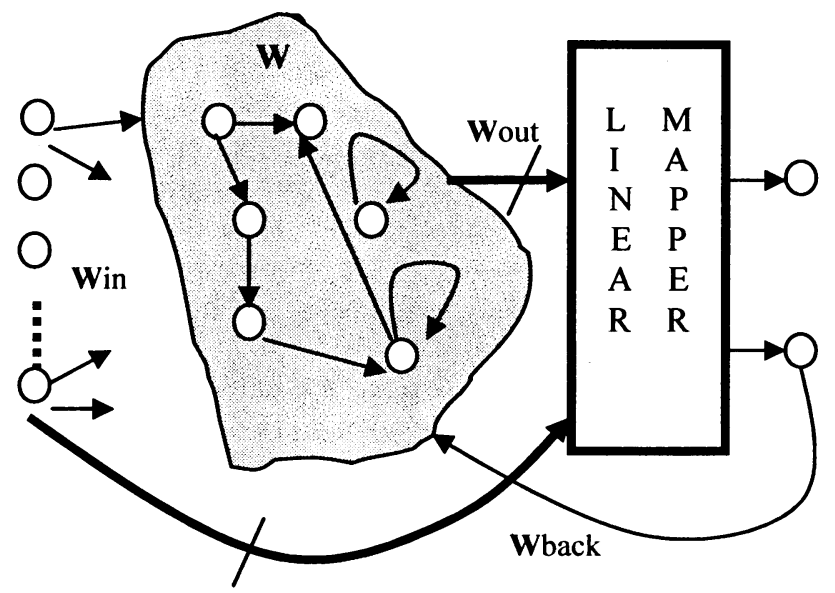

Figure 1. The block diagram of an echo state network 
The paper is organized as follows. In section II, we give a brief overview of ESNs with the echo state condition. Section III introduces transiently stable computation with echo state networks. The principles of transiently stable computation are discussed in section IV. Section V concludes the paper.

\section{OVERVIEW OF ECHO STATE NETWORKS}

RNNs are powerful dynamical neural systems that are able to process signals with temporal structure. The recurrent connections between PEs allow the system to incorporate input dynamics in the system response without the use of explicit delays at the front end as is done in TDNNs. On the other hand, it is the same recurrent structure that leads to difficulties in training RNNs since the gradient information is not available instantaneously but rather distributed over time [2]. Any gradient-based training algorithm designed for RNNs with simple sigmoid nonlinearities suffers from the problem of gradient decay through time and topology resulting in slow training and increased computational complexity. Moreover, the performance surfaces are complex including many local minima and the system can easily become unstable during training because of the recurrent connections [2].

The ESN idea intends to overcome these difficulties by fixing $a$ priori the recurrent network connections and adapting only an instantaneous readout network [3] (see Fig. 1). The echo state at time $n, x(n)$, is calculated according to

$$
\mathbf{x}(n+1)=\mathbf{f}\left(\mathbf{W}^{i n} \mathbf{u}(n+1)+\mathbf{W} \mathbf{x}(n)+\mathbf{W}^{\text {back }} \mathbf{y}(n)\right)
$$

The network output, $y(n)$, is given by

$$
\mathbf{y}(n+1)=\mathbf{f}^{\text {out }}\left(\mathbf{W}^{\text {inout }} \mathbf{u}(n+1)+\mathbf{W}^{\text {out }} \mathbf{x}(n+1)+\mathbf{W}^{\text {outout }} \mathbf{y}(n)\right)
$$

Here, $\mathbf{u}(n)$ is the input signal and $\mathbf{W}^{\text {in }}, \mathbf{W}, \mathbf{W}^{\text {back }}, \mathbf{W}^{\text {inout }}, \mathbf{W}^{\text {out }}$ and $\mathbf{W}^{\text {outout }}$ are the matrices defining the connection weights between the processing elements. The nonlinear function $\mathbf{f}($.) is chosen to be sigmoid hyperbolic tangent function, $\tanh ($.$) ,$ whose derivative is maximum with a value of 1 at operating point zero. For details of ESN mathematics, refer to [3].

The fixed connection weights of the ESN are chosen with the help of the echo state condition $(|\mathbf{W}|<1)$, which is derived from the stability of the linearization of the ESN around zero equilibrium [3]. This condition guarantees the asymptotic stability of zero state for autonomous system. In practice, $\mathbf{W}$ is chosen to be a random sparse matrix, to avoid too much dependency (correlation) between the states. Designing the $\mathrm{W}$ matrix using an information theoretic criterion on the states has been proposed in [5], yet this approach also obeys the echo state condition.

A simple demonstration of the typical response of an ESN with echo state condition is given in Fig. 2. A randomly connected 100-unit ESN without output feedback connections is constructed. The entries of the internal connection weight matrix $\mathrm{W}$ are set to $0.4,-0.4$ and 0 with probabilities of $0.025,0.025$ and 0.95 resulting in a spectral radius of 0.9 . The input weight matrix, $W^{\text {in }}$ has values +1 or -1 with equal

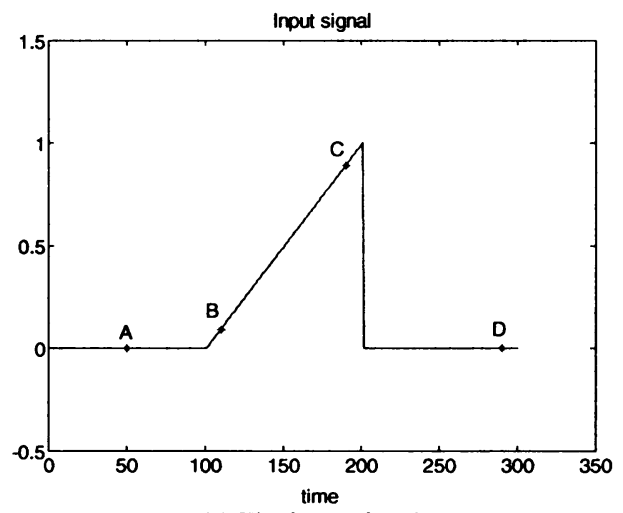

(a) The input signal

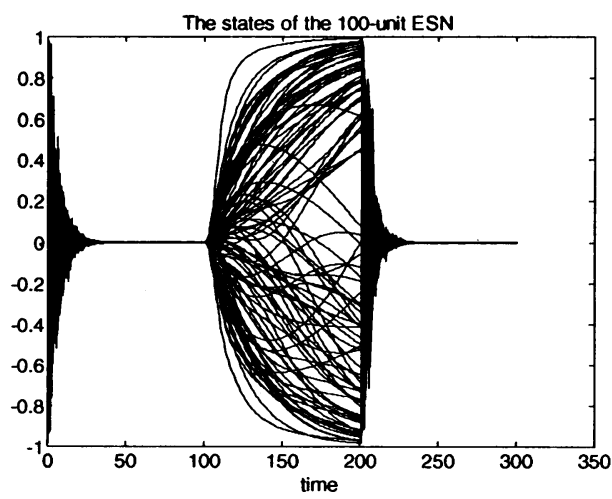

(b) 100 echo states of ESN

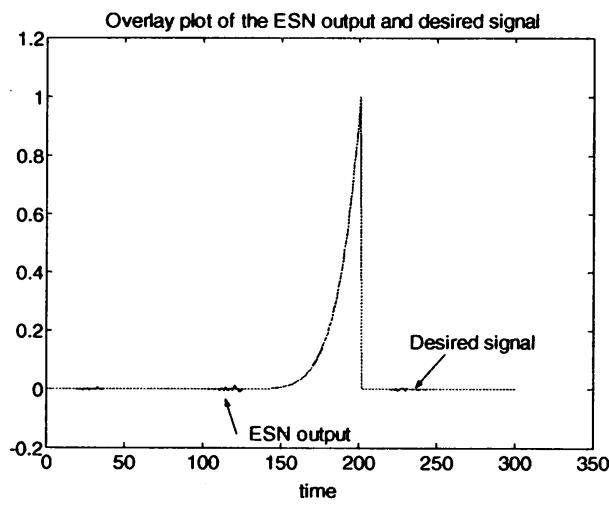

(c)The overlay plot of the ESN output and the desired signal

Figure 2. Demonstration of a typical response of ESN with a spectral radius of 0.9 and an application for function approximation

probabilities. The input to the system has 3 different regions, a zero signal for the first 100 steps, a ramp signal for the following 100 steps and again a zero signal of length 100 (Fig. 2a). The system is initialized randomly and run with the given input. Fig. $2 b$ depicts all the 100 echo states on top of each other over the given time interval. As seen from the figure, during the first 100 steps, the system state converged to zero (after an initial transient of length about 30 steps caused by the random initial condition) since the input to the system is zero and the spectral radius is less than 1 . Then, the echo states are constructed by the ramp signal between the time interval 100 and 200 . The states again converge to zero due to the echo state condition, when the input is removed. 
According to [5], the echo states form a set of basis functions that are derived dynamically from the history of the input signal. The instantaneous readout linearly combines these basis functions in order to generate a given desired response. Here, the resulting echo states will be used to generate the desired response that is chosen to be the seventh power of the input signal. The readout weights, optimal in the mean square error sense (MSE), can be calculated using the well-known Wiener-Hopf equation (since the readout is a static linear mapper) given by

$$
\mathbf{W}^{\text {out }}=E\left[\mathbf{x} \mathbf{x}^{T}\right]^{-1} E[\mathbf{x d}] \cong\left(\frac{1}{N} \sum_{n} \mathbf{x}(n) \mathbf{x}(n)^{T}\right)^{-1}\left(\frac{1}{N} \sum_{n} \mathbf{x}(n) \mathrm{d}(n)\right)
$$

Fig. 2c displays the overlay plot of the optimal ESN output and the desired signal. As observed from the figure, the system output very accurately estimates the desired signal.

\section{TRANSIENTLY STABLE COMPUTATION WITH ECHO STATE NETWORKS}

Engineering systems are mostly designed with a global stability restriction. We have seen a similar constraint, echo state condition, in the design of echo state networks. We have also demonstrated with a function approximation experiment that ESNs with the echo state condition can do useful computation. On the other hand, biological computation may not possess a similar stability constraint [6]. Indeed, biological systems do not have fixed point dynamics but rather wide variety of collective behavior in the form of oscillations and even chaos [6] [7].

Inspired from principles of biological computation, we would like to explore the network's response without the restriction on the spectral radius of $\mathbf{W}$. Hence, we will remove the echo state condition and let the spectral radius to be slightly greater than 1 . Obviously, this will introduce instability for the autonomous system whereas the response of the system with an applied input is yet not clear.

In order to observe the ESN response without the echo state condition, we will use a similar experimental set up that we used in section II. We again use a 100-unit ESN without output feedback connections. The entries of $\mathrm{W}$ matrix are chosen from the same weight distribution as in section II but they are scaled such that a spectral radius of 1.1 is obtained. The same input signal (see Fig. 2a) is fed to the 100-unit ESN that is initialized to a random initial condition. We plot the resulting echo states in Fig. 3a. There are a few observations to be made at this point. First of all, we see that the system does not converge to zero during the first 100 steps although the input is zero. Instead, the echo states exhibit a nonconvergent dynamical response that differs from the ESN with the echo state condition. In fact, this was the expected response, since the system is designed to be unstable (spectral radius is greater than one) around zero equilibrium. A similar response can also be observed during the last 100 time steps when the input is again zero. The second and more important observation is the response of the system between time steps 100 and 200 where the ramp signal is applied as the input.

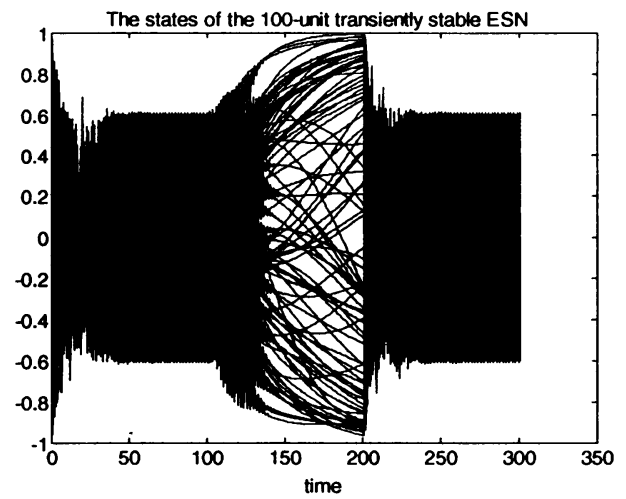

(a) 100 echo states of the transiently stable ESN

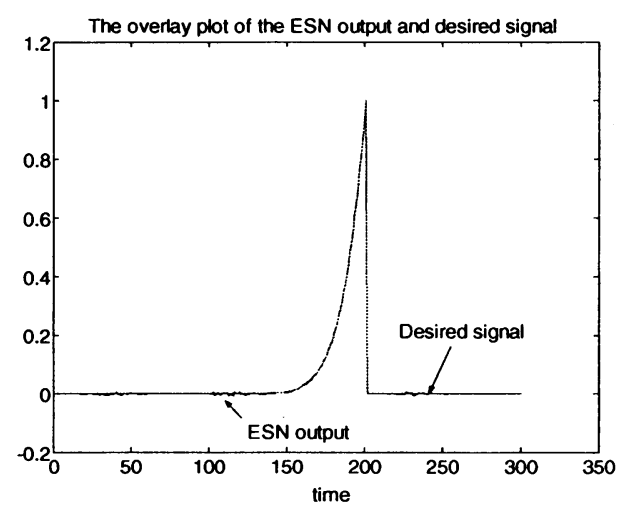

(b)The overlay plot of the ESN output and the desired signal

Figure 3. Demonstration of a typical response of transiently stable ESN with a spectral radius of 1.1 and an application for function approximation

The echo states during this interval become more regular (after some initial transient between time steps 100 and 130) compared to the states when there is no input. Moreover, after the transient, the echo states look similar to the ones in Fig. $2 \mathrm{~b}$ where the ESN satisfies the echo state condition. Thirdly, there is a transition period between time steps 100 and 130 where the states switch from a disordered mode to a more regular mode. In summary, the system responds according to its own dynamics when there is no input. As the input amplitude gradually increases, the system response is determined by a competition between the system dynamics and the input amplitude. When the input amplitude is sufficiently large, the system dynamics becomes "transiently stable" and is determined by the input signal.

We would like to find out if we can utilize the transiently stable ESN for the same function approximation problem we had in section II. The weights of the linear readout network are again computed using (3) and the corresponding output is generated in Fig. 3b. As seen from the figure, we got a good match between the output and the desired signal. Similar results can be obtained even if the system is initialized to a different initial condition or the time instant at which the ramp signal is applied (this will change the state value when the ramp signal is applied) changes. This experiment clearly 
demonstrates that "transiently stable" ESN can do useful computation.

\section{UNDERSTANDING COMPUTATION WITH TRANSIENTLY STABLE STATES}

In the previous section, we have observed that the response of the transiently stable ESN is determined by an intrinsic competition between the system dynamics and the input signal. We have discovered the change in the local dynamics of the system from a disordered state into a more regular one with the application of the input signal. Hence, we will quantify the local dynamics of the system obtained from the linearization of the nonlinear ESN, as is done in [5], in order to better understand the principles of computation in transiently stable systems.

The dynamics of a nonlinear system can be approximated by that of a linear system in a small local neighborhood of a given equilibrium point [9]. Here, we will approximate the ESN dynamics by the dynamics of the linearized system in the local neighborhood of the current value of the system state. Hence, when the system operating point varies over time, the linear system approximating the ESN dynamics will change.

Consider the update equation for the echo states given in (1) without the output feedback connections ( $\mathbf{W}^{\text {back }}$ is zero). We can linearize the system around the current state, $\mathbf{x}(n)$ and obtain the Jacobian matrix, $\mathbf{J}$, defined by

$$
\begin{aligned}
J & =\left[\begin{array}{cccc}
\dot{f}\left(x_{1}\right) w_{11} & \dot{f}\left(x_{1}\right) w_{12} & \ldots & \dot{f}\left(x_{1}\right) w_{1 N} \\
\dot{f}\left(x_{2}\right) w_{21} & \dot{f}\left(x_{2}\right) w_{22} & \ldots & \dot{f}\left(x_{2}\right) w_{2 N} \\
\ldots & \ldots & \ldots & \ldots \\
\dot{f}\left(x_{N}\right) w_{N 1} & \dot{f}\left(x_{N}\right) w_{N 2} & \ldots & \dot{f}\left(x_{N}\right) w_{N N}
\end{array}\right] \\
& =\left[\begin{array}{cccc}
\dot{f}\left(x_{1}\right) & 0 & \ldots & 0 \\
0 & \dot{f}\left(x_{2}\right) & \ldots & 0 \\
\ldots & \ldots & \ldots & \ldots \\
0 & 0 & \ldots & \dot{f}\left(x_{N}\right)
\end{array}\right] \cdot \mathbf{W}
\end{aligned}
$$

In (4), $x_{i}$ denotes the $i^{\text {th }}$ entry of the current state vector and $w_{i j}$ denotes the $(i, j)^{\text {th }}$ entry of $\mathbf{W}$ matrix. Here, we are particularly interested in the movement of the poles of the linear systems, since poles determine the stability of the system. The largest slope is at the origin; therefore the $J$ matrix has the largest spectral radius. But in doing so the mode that is unstable will pump energy and the increased amplitude in the states will visit areas of the nonlinearity with smaller slopes which will decrease the spectral radius of the weight matrix. This effectively will stabilize the system dynamics as can be seen in Fig 3a, where the amplitude of the oscillation is less than the full dynamic range of the ESN. The process will continue with the poles moving around in closed trajectories. Therefore the ESN with sigmoid nonlinearity has a built in mechanism to avoid hitting the rails of the nonlinearities and produce very complex signal time structure.

First, we will investigate the movement of the poles for the ESN given in section II with the same input signal. A similar analysis has been done in [5] with a sinusoidal input. Fig. 4 shows the pole locations at different time instances. Similar to the conclusions in [5], we see that the poles always stay inside the unit circle even though the input signal changes. Secondly, after the states converge to zero, the poles stay still until the ramp signal arrives. Moreover, as the input signal strengthens, the poles move towards the origin of the z-plane (decreases the spectral radius), which results in a more stable system. This is due to the fact that the higher input signals saturate the nonlinear function reducing the slope at which the system is operating.

Now, let us examine the movement of poles of the transiently stable ESN. The spectral radius is set to 1.1 keeping all the other parameters of the ESN unchanged. Fig.5 shows the movement of the poles for the same input signal. First of all, the poles of the system move in and out of the unit circle during the first and last 100 time steps (hence zero state is not asymptotically stable). This explains the complex echo state signal when there is no input to the system (see fig. $3 b$ ). The poles of the ESN with spectral radius 0.9 stays inside the unit circle during the same time interval. Secondly, when the ramp input is applied, the poles start to shrink towards the unit circle (after some transient) as is observed in the stable ESN. This phenomenon is again due to the sigmoid nature of nonlinearity, which saturates with large input signals reducing the slope of the operating point in (4). With the movement of the poles towards the origin, the system stabilized transiently allowing the system state to be controlled by the input. This is critical for the use of the system for computation (function approximation). Finally, we observe a transient time, when the ramp signal is applied, before the poles start shrinking. In this period, there is a competition between the system dynamics and the input signal.

There are a few extra comments about the selection of the spectral radius to be made at this point. First of all, the transient response time after the ramp is applied is determined by two factors, the spectral radius and the slope of the ramp signal. The transient time will increase if the spectral radius of $\mathrm{W}$ is increased or the slope of the ramp signal is reduced. Because as the spectral radius is increased, the force required to bring the poles inside the unit circle is increased, hence a larger signal is required. Therefore, there is a balance between the system dynamics determined by the spectral radius and the input signal required to stabilize the system. Secondly, it is important to quantify the unstable behavior of the signals present in the transiently stable ESN when the input is absent. We have observed that when the spectral radius is slightly greater than 1 , the signals are periodic oscillations whereas for larger values of the spectral radius, the signals are more irregular and even chaotic. 


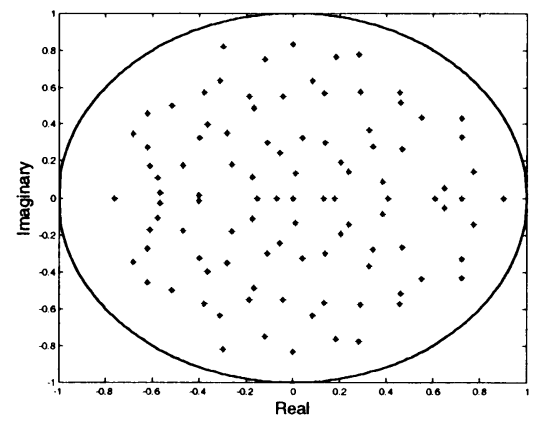

(a)

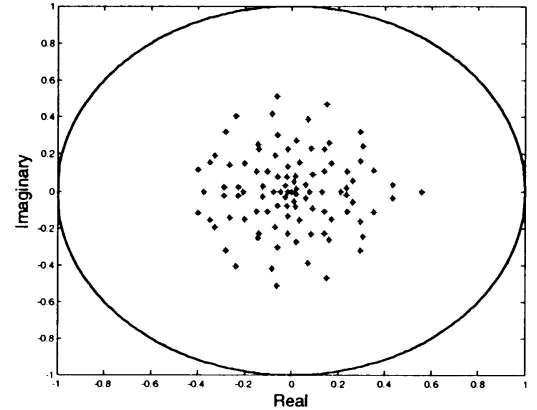

(c)

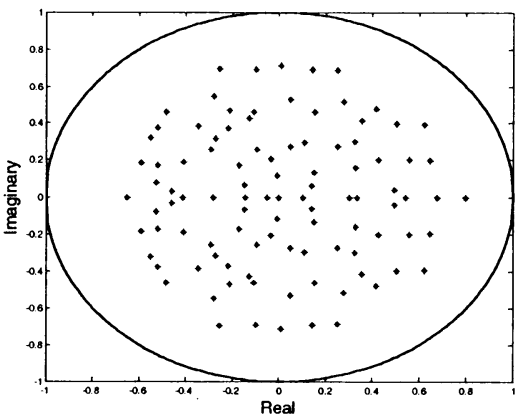

(b)

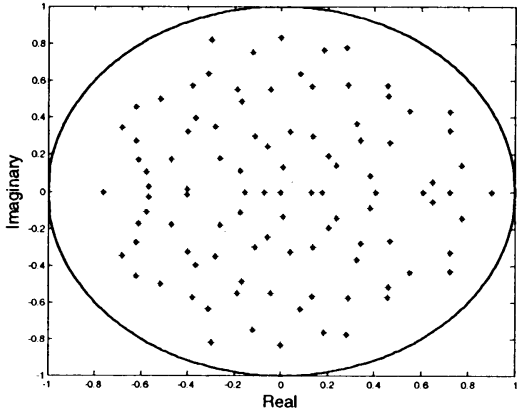

(d)

Figure 4. The distribution of the poles for ESN with spectral radius 0.9 are plotted in figures a, b, c, $\mathrm{d}$, when the input is at points labeled by A, B, C, D in figure $2 \mathrm{a}$, respectively

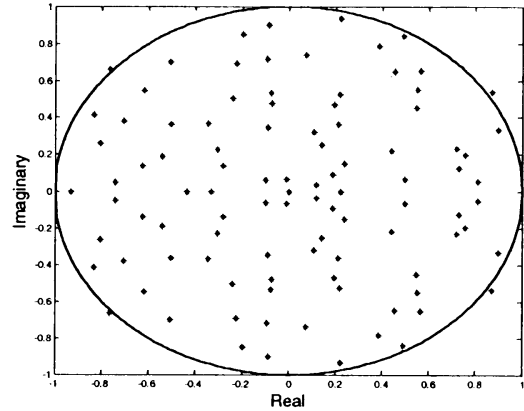

(a)

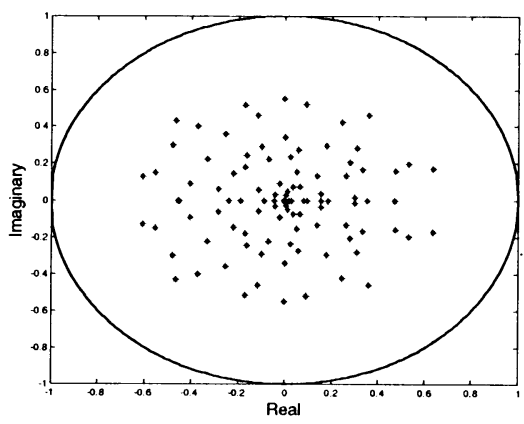

(c)

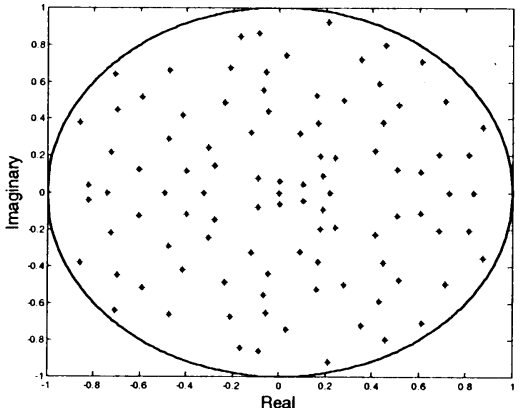

(b)

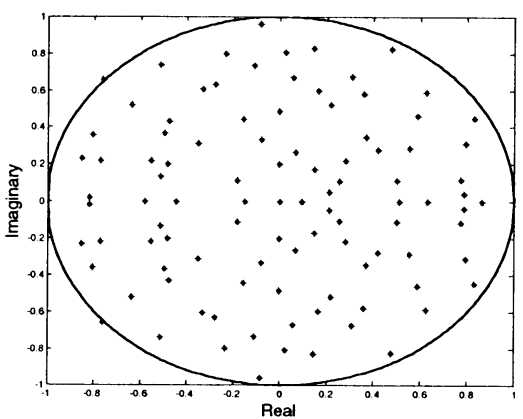

(d)

Figure 5. The distribution of the poles for ESN with spectral radius 1.1 are plotted in figures a, b, c, d, when the input is at points labeled by A, B, C, D in figure 2a, respectively 


\section{CONCLUSIONS}

Linear system theory shows clearly that stability is necessary to obtain useful responses from linear systems. However, this argument does not necessarily apply to nonlinear systems as demonstrated in this paper. A nonlinear system can be unstable and still be able to have dynamics that are controlled by the input, when it is applied. Hence the title of the paper, computation with transiently stable states.

It is too early to judge the importance of this regime for computation. Indeed, we found that the system still performs well in function approximation, but what are the advantages with respect to the regime of $|\mathrm{W}|<1$ ? We need to move to more complex paradigms of computation to see any advantage for the transient stable regime. Firstly, the transiently stable regime resembles the "edge of chaos" computation [8]. Indeed, during the initial states of the ramp, the system is marginally unstable, which is one of the characteristics of the edge-of-chaos. But instead of being studied in cellular automata, we now have an ESN performing computation that we can model, understand and utilize (function approximation). Secondly, from a computation point of view, this regime is interesting because it toggles between internal dynamics and external dynamics. It may be possible to have the external dynamics control the initial conditions for the internal dynamics and therefore be able to move to very different parts of the state space (memories) as proposed by Freeman in his olfactory model, and recognize different stimuli [7]. We will investigate this further in a follow up paper.

Acknowledgements: This work was partially supported by NSF grant EIA 0135946.

\section{REFERENCES}

[1] T. Kailath, Linear systems, Prentice Hall, Englewood Cliffs, NJ, 1980.

[2] S. Haykin, Neural Network: A comprehensive Foundations, Prentice Hall, Englewood Cliffs, NJ, 1998.

[3] H. Jaeger, "The echo state approach to analyzing and training recurrent neural networks," Technical Report GMD Report 148, German National Research Center for Information Technology.

[4] H. Jaeger, "Short term memory in echo state networks," Technical Report GMD Report 152, German National Research Center for Information Technology.

[5] M. C. Ozturk, D. Xu, J. C. Principe, "Analysis and Design of Echo State Networks for Function Approximation," submitted to Neural Computation

[6] Y. Yao, W.J. Freeman, "Model of Biological Pattern Recognition and Spatially Chaotic Dynamics," Neural Networks, vol.3, pp. 153-170, 1990.

[7] Y. Yao, W.J. Freeman, B. Burk, "Central Pattern Generating and Recognizing in Olfactory Bulb: A Correlation Learning Rule," Neural Networks, vol. 1, pp. 277-288, 1988.

[8] C. G. Langton, "Computation at the Edge of Chaos: phase transitions and emergent computation," Physica D, vol.42, pp. 12-37, 1990.

[9] Y. Kuznetsov, L. Kuznetsov, J. Marsden, Elements of Applied Bifurcation Theory, Prentice Hall, SpringerVerlag, New York, 1998. 